\title{
Time Scales for Viscous Flow, Atomic Transport, and Crystallization in the Liquid and Supercooled Liquid States of $\mathrm{Zr}_{41.2} \mathrm{Ti}_{13.8} \mathrm{Cu}_{12.5} \mathrm{Ni}_{10.0} \mathrm{Be}_{22.5}$
}

\author{
A. Masuhr, T. A. Waniuk, R. Busch,* and W. L. Johnson \\ W. M. Keck Laboratory of Engineering Materials, California Institute of Technology, Pasadena, California 91125
}

(Received 25 September 1998)

\begin{abstract}
The shear viscosity of liquid $\mathrm{Zr}_{41.2} \mathrm{Ti}_{13.8} \mathrm{Cu}_{12.5} \mathrm{Ni}_{10.0} \mathrm{Be}_{22.5}$ has been measured. At the liquidus temperature we find an extremely high viscosity of $2.5 \mathrm{Pas}$, favoring glass formation. At deep supercooling the time scales for the diffusion of small and medium sized atoms as reported in the literature decouple from the internal relaxation time as probed by our viscosity measurements. Similarly, crystallization from the supercooled liquid state can be described with an effective diffusivity that scales with the viscosity at high temperatures and is Arrhenius-like at deep supercooling. [S00319007(99)08631-7]
\end{abstract}

PACS numbers: 61.43. $-\mathrm{j}, 64.70 . P f, 66.10 . \mathrm{Cb}, 66.20 .+\mathrm{d}$

Monatomic and binary metallic liquids at the melting point have viscosities $\eta$ of about $10^{-3}$ Pas and diffusivities on the order of $10^{-9} \mathrm{~m}^{2} \mathrm{~s}^{-1}$ [1]. Their temperature dependencies can be described by apparent activation energies $Q$ of less than $0.5 \mathrm{eV}[2]$. By containerless processing or fluxing techniques, these liquids may be supercooled below their melting points, but critical cooling rates to bypass crystallization and form a glass are typically $10^{7}$ to $10^{9} \mathrm{~K} \mathrm{~s}^{-1}$.

Recently, diffusion [3-6] and viscosity measurements [7-9] as well as crystallization studies [10-12] in the deeply supercooled liquid of multicomponent bulk metallic glass (BMG) forming alloys have found considerable attention. Still, only limited data are available for transport coefficients in the equilibrium or slightly supercooled liquid of BMG forming alloys. However, the temperature range between the liquidus temperature, $T_{\text {liq }}$, and the temperature with the minimum time to crystallization, $T_{n}$, is decisive for the glass forming ability of a liquid upon constant cooling [13]. In the following we will present results from viscosity measurements and crystallization studies on $\mathrm{Zr}_{41.2} \mathrm{Ti}_{13.8} \mathrm{Cu}_{12.5} \mathrm{Ni}_{10.0} \mathrm{Be}_{22.5}$ (V1) and discuss the time scales for viscous flow and crystallization along with published results on atomic transport.

We have designed a high temperature Couette viscometer to measure rheological properties of Zr-based glass forming alloys in the viscosity range from $10^{-2} \mathrm{Pas}$ to $10^{3} \mathrm{Pas}$. The viscometer's concentric cylinder shear cell is machined from graphite and is mounted vertically inside a high vacuum induction furnace. The outer cylinder is attached to a static torque sensor and the temperature is measured with a thermocouple mounted inside the cylinder wall. Spatial temperature variations within the shear cell are less than $\pm 4 \mathrm{~K}$ as observed by optical pyrometry. Under continuous rotation of the inner cylinder the static torque on the outer cylinder is proportional to the viscosity of the liquid. The proportionality constant can be calculated from the rotational frequency and the geometry of the shear cell [14].
Results obtained from the high temperature viscometer as well as from beam bending rheometry [9] are summarized in Fig. 1. At the liquidus temperature, $T_{\text {liq }}=$ $1026 \mathrm{~K}$, we find a viscosity of $2.5 \mathrm{~Pa}$ s with a slope [2] of $2.0 \mathrm{eV}$, both of which are extraordinary high values for a metallic system. Phenomenological models based on entropy or free volume can be used to describe the viscosity data [15]. Within the free volume model the viscosity,

$$
\eta=\eta_{0} \exp \left(b \nu_{m} / \nu_{f}\right)
$$

is expressed in terms of $\nu_{f}$, the average free volume per atom, and $b \nu_{m}$, the critical volume for flow [16]. The

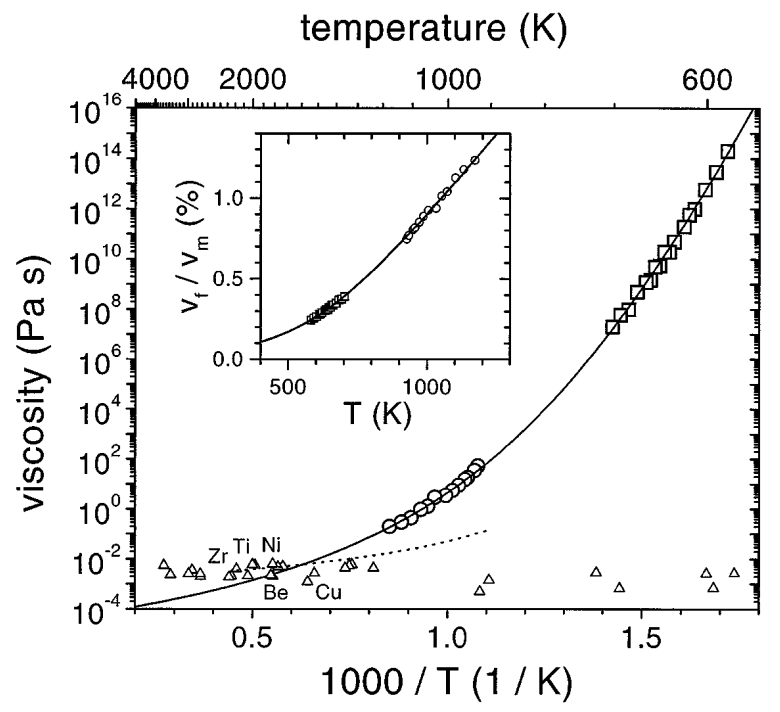

FIG. 1. Viscosity of V1 as measured by beam bending ( $\square$ ) and concentric cylinder viscometry $(\bigcirc)$ as well as a leastsquares fit (-) to the free volume model, Eqs. (1) and (2). Melt viscosities $(\triangle)$ of $\mathrm{T} 1, \mathrm{Cd}, \mathrm{Pb}, \mathrm{Zn}, \mathrm{Te}, \mathrm{Sb}, \mathrm{Mg}, \mathrm{Ag}, \mathrm{Ac}$, $\mathrm{Au}, \mathrm{Cu}, \mathrm{Mn}, \mathrm{Be}, \mathrm{Ni}, \mathrm{Co}, \mathrm{Fe}, \mathrm{Sc}, \mathrm{Pd}, \mathrm{V}, \mathrm{Ti}, \mathrm{Pt}, \mathrm{Zr}, \mathrm{Cr}, \mathrm{Rh}, \mathrm{B}$, $\mathrm{Ru}$, Ir, Mo, Os, Re, and $\mathrm{W}$ near their melting point (in order of increasing melting point) [1] and an extrapolation (---) of the viscosity of $\mathrm{Ni}$ into its supercooled liquid state. Free volume of V1 as calculated from the viscosity data and Eqs. (1) and (2) (inset).

(C) 1999 The American Physical Society 
atomic volume $\nu_{m}$ of $\mathrm{V} 1$ was measured to be $1.67 \times$ $10^{-29} \mathrm{~m}^{3}$ near the liquidus [17]. The prefactor, $\eta_{0}=$ $h / \nu_{m}$, in Eq. (1) is set to $4 \times 10^{-5} \mathrm{Pas}$, where $h$ is Planck's constant [18]. A least-squares fit of the logarithm of the viscosity data to Eq. (1) with a temperaturedependent free volume [16],

$$
\nu_{f}=\frac{k}{2 \varsigma_{0}}\left(T-T_{0}+\sqrt{\left(T-T_{0}\right)^{2}+\frac{4 \nu_{a} \varsigma_{0}}{k} T}\right),
$$

according to Cohen and Grest (CG) describes the data very well, as shown in Fig. 1. The three fit parameters are found to be $b \nu_{m} \varsigma_{0} / k=4933 \mathrm{~K}$ [19], $T_{0}=672 \mathrm{~K}$, and $4 \nu_{a} \varsigma_{0} / k=162 \mathrm{~K}$.

The CG model of a volume dependent free energy for liquidlike and solidlike cells that led to Eq. (2) describes the present viscosities better than a Vogel-FulcherTammann (VFT) relation [15]. The transition region from $\nu_{f} \sim\left(T-T_{0}\right)$ for large $T$ to $\nu_{f} \sim T$ for small $T$, i.e., from VFT to Arrhenius behavior, is found to be about $T_{0}=672 \mathrm{~K}$. In contrast to the VFT equation (see, e.g., $[7,8,20])$, the viscosity remains well defined below $T_{0}$.

The high temperature viscosity data yield $\alpha_{f} / b=$ $1.83 \times 10^{-4} \mathrm{~K}^{-1}$ with $\alpha_{f}$ as the average expansion coefficient for the free volume. This increase in free volume with temperature can be approximated by the difference between the thermal expansion coefficients of the liquid and the glass, $\alpha_{f}=\alpha_{\text {liq }}-\alpha_{\text {glass }}[17,21]$, yielding $b=0.105$ and an average free volume per atom, $\nu_{f} / \nu_{m}$, as shown in Fig. 1 . The small amount of free volume at the liquidus, $\nu_{f}\left(T_{\text {liq }}\right)=9.6 \times 10^{-3} \nu_{m}$, is consistent with the picture of a dense metallic liquid with slow kinetics. Extrapolating the viscosity of V1 to higher temperatures (see Fig. 1) one finds a normal melt viscosity for a metallic system at about the average melting point of V1's constituents.

At temperatures below $650 \mathrm{~K}$, the isothermal relaxation of the viscosity of $\mathrm{V} 1$ to its equilibrium values has been measured [9]. Similar to the viscosity relaxation in $\mathrm{Zr}_{46.75} \mathrm{Ti}_{8.25} \mathrm{Cu}_{7.5} \mathrm{Ni}_{10} \mathrm{Be}_{27.5}$ (V4) [20], the internal equilibration times $\tau_{\eta}$ associated with this relaxation into the supercooled liquid state are found to be proportional to the viscosity: $\tau_{\eta}=\eta / G_{\eta}$. The experimental values of $\tau_{\eta}$ and $\eta$ yield $G_{\eta}=5.5 \times 10^{8} \mathrm{~Pa}$, where we have neglected the temperature dependence of $G_{\eta}$ compared to $\eta$ and $\tau_{\eta}$. The times $\tau_{\eta}$ in Fig. 2 are in good agreement with the total times for the calorimetric glass transition upon linear heating with rate $R$. From differential scanning calorimetry we define the latter by $\left(T_{g}^{\text {end }}-\right.$ $\left.T_{g}^{\text {onset }}\right) / R$ [22]. Note that $\tau_{\eta}$ is proportional but not equal to the relaxation time as defined within Maxwell's model of viscoelasticity. From the shear modulus of V1 of $G=3.3 \times 10^{10} \mathrm{~Pa}[23]$ we expect the Maxwell time $(\eta / G)$ for shear stress relaxation after small deformations to be about 2 orders of magnitude faster than the internal equilibration time $\tau_{\eta}$.

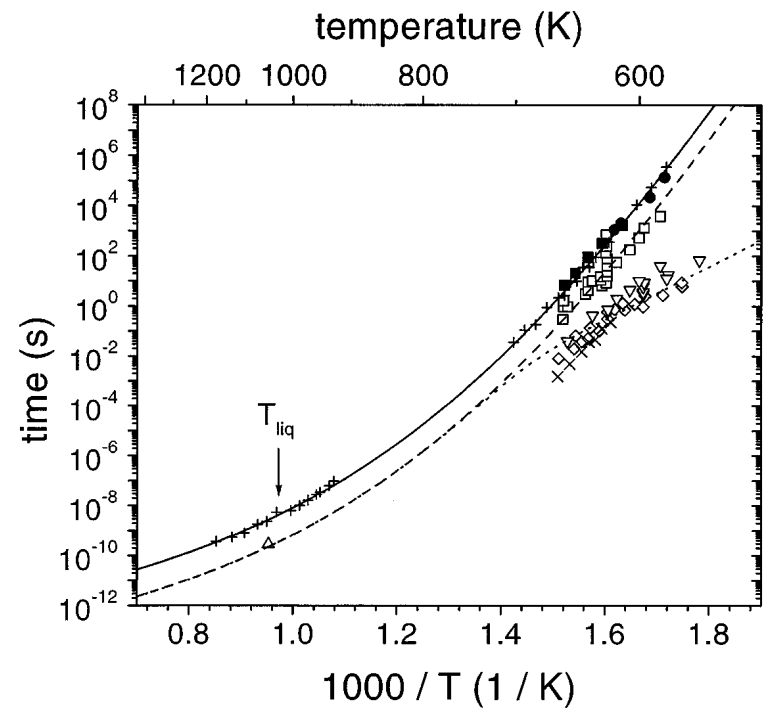

FIG. 2. Structural relaxation times $\tau_{\eta}(\mathbf{O})$ and $\tau_{\eta}=\eta / G_{\eta}$ from equilibrium viscosities (+) of $\mathrm{V} 1$ and fit to Eq. (3) (-). Total times for calorimetric glass transition ( $\boldsymbol{\square})$ from differential scanning calorimetry upon linear heating. Characteristic diffusion times, $\tau_{D}$, for $\mathrm{Be}(\times)$ [3], Ni $(\diamond)[4]$, Co $(\nabla), \mathrm{Al}(\square)$ [5], and $\mathrm{Au}(\triangle)$ [24] in V1. Hybrid model for $\mathrm{Al}(--)$ and $\mathrm{Ni}$ $(\cdots)$ diffusion (see text).

Recently, studies of Be interdiffusion and $\mathrm{Ni}, \mathrm{Co}$, and $\mathrm{Al}$ radiotracer diffusion in deeply supercooled V1 were performed [3-5]. Using the equation for the random walk of the $i$ th tracer, $\tau_{D, i}=l^{2} / 6 D_{i}$, we consider times, $\tau_{D, i}$, for the successful displacement of an average atomic diameter $l$. This definition of $\tau_{D, i}$ is not connected to a particular diffusion mechanism. From the atomic volume $\nu_{m}, l$ is calculated to be $3.2 \times 10^{-10} \mathrm{~m}$ and the corresponding $\tau_{D, i}$ are added in Fig. 2. At about $600 \mathrm{~K}$ the diffusivities of $\mathrm{Al}$ and Ni differ by 3 orders of magnitude, while they show a tendency to merge at higher temperatures. The temperature dependence of $\mathrm{Al}$ diffusion is similar to that of the viscosity: $\tau_{D, \mathrm{Al}} \cong \tau_{\eta} / 14$ (dashed curve in Fig. 2). Ni and Co show significantly smaller absolute values of $\tau_{D}$ and activation energies of about 2.0 to $2.2 \mathrm{eV}$. Clearly, the time scales for viscous flow and diffusion of medium sized atoms have different temperature dependencies in the deeply supercooled liquid state. A collective hopping mechanism is likely to control the diffusion of $\mathrm{Ni}$ and $\mathrm{Co}$ in this temperature range as this behavior has been confirmed for Co in V4 by isotope effect measurements [6]. Similarly, we expect the migration of the smallest atom in the alloy, Be, to also be controlled by thermally activated jumps on a potential energy surface that fluctuates on a time scale given by $\tau_{\eta}$.

For the equilibrium liquid, only preliminary results of $\mathrm{Au}$ interdiffusion with the equilibrium melt of $\mathrm{V} 1$ at $1050 \mathrm{~K}$ are available [24]. Quasielastic neutron scattering experiments on V4 revealed that above the liquidus temperature the differences in the diffusion coefficients of the various elements in the liquid alloy are less than 1 
order of magnitude [25]. This finding is in accordance with the merging tracer diffusion coefficients in Fig. 2 and is well known for foreign diffusion in the equilibrium melts of $\mathrm{Ag}, \mathrm{Hg}, \mathrm{Sn}, \mathrm{Pb}$, and $\mathrm{Bi}$ [26].

Guided by the differences in diffusion of the various elements at low temperatures and by the scaling between viscosity and diffusion in the equilibrium liquid, we consider a hybrid equation for the diffusion times $\tau_{D}$ of medium sized atoms in V1. Within this model, the time for a successful displacement $l$ of, e.g., Ni can either be limited by density fluctuations of the matrix or by a solidlike jump, where Eq. (3) takes into account the parallel development of both processes,

$$
\frac{1}{\tau_{D, \mathrm{Ni}}}=\frac{g_{\mathrm{Ni}}}{\tau_{\eta}}+\frac{1}{\tau_{\mathrm{Ni}, 0}} \exp \left(-\frac{Q_{\mathrm{Ni}}}{k T}\right) .
$$

At high temperatures, $\tau_{D, \mathrm{Ni}}$ is limited by the first term on the right-hand side of Eq. (3), i.e., by the time scale for changes in the surrounding matrix, $\tau_{\eta}$. The value of $g_{\mathrm{A} 1}=14$ (see above) may serve as a lower limit and a first estimate for $g_{\mathrm{Ni}}$ as we do not expect $\mathrm{Ni}$ diffusion to be faster than $\mathrm{Au}$ near the liquidus (see Fig. 2). The corresponding times, $\tau_{D, \mathrm{Ni}}$, are plotted in Fig. 2 with $Q_{\mathrm{Ni}} \cong 2.1 \mathrm{eV}$ (dotted curve). Below about $800 \mathrm{~K}$, thermally activated jumps of $\mathrm{Ni}$ with an average activation energy $Q_{\mathrm{Ni}}$ can occur as the potential energy barriers for $\mathrm{Ni}$ diffusion become fixed during the time between two successive jumps. Both processes in Eq. (3) act in parallel in a relatively wide intermediate temperature range between about 700 to $800 \mathrm{~K}$, which can be regarded as the glass transition regime for Ni diffusion in V1. This temperature range is independent of the time scale of the experiment but may differ among the various components in the alloy. In fact, for the fast diffusion species $(\mathrm{Ni}, \mathrm{Cu}$, $\mathrm{Be}$ ) in the alloy we expect a cascade of transitions. The jumps of a medium sized atom, such as $\mathrm{Ni}$ in the dense packed supercooled liquid, will probably also affect the positions of the fastest diffusor, Be.

Starting from the equilibrium liquid, we studied the temperature dependence of the onset of crystallization of V1 under isothermal conditions over the entire supercooled liquid range (see [14]). The logarithm of the onset times $t_{x}$ of the first recalescence event as observed in the thermocouple signal are shown in Fig. 3. The times to crystallization show a minimum at $T_{n}=895 \mathrm{~K}$, due to the competition between an increasing thermodynamic driving force for crystallization and decreasing atomic mobility upon supercooling. The shortest times to crystallization are as large as $60 \mathrm{~s}$ at this temperature, reflecting a critical cooling rate for glass formation of only $1 \mathrm{~K} / \mathrm{s}$. This rate is at least 6 orders of magnitude smaller than values for simple metallic systems. At the temperature $T_{n}$, the viscosity of $\mathrm{V} 1$ is about 4 orders of magnitude larger than the viscosity of, e.g., $\mathrm{Ni}$ at a similar degree of supercooling (see Fig. 1). We conclude that the sluggish kinetics at elevated temperatures discussed above account

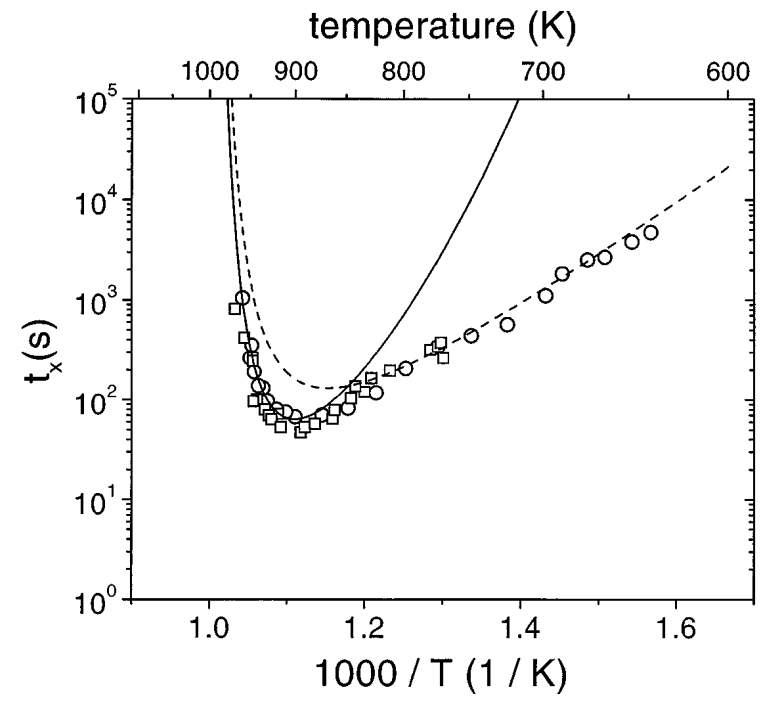

FIG. 3. Onset of recalescence under isothermal conditions in the supercooled liquid state of V1 from electrostatic levitation ( $\square$ ) [27] and from processing in graphite crucibles ( $\bigcirc$ ). Calculated times for a crystalline volume fraction of $x=10^{-4}$ using $D_{\text {eff }} \sim \eta^{-1}(-)$ and $D_{\text {eff }} \sim \exp \left(-Q_{\text {eff }} / k T\right)(--)$.

for a significant part of V1's exceptional glass forming ability.

Within classical nucleation theory the steady state nucleation rate is given by $I_{s}=A D_{\text {eff }} \exp \left(-\Delta G^{*} / k T\right)$, where $D_{\text {eff }}$ is an effective diffusivity and $\Delta G^{*}=$ $16 \pi \sigma^{3} / 3 \Delta G^{2}$. For the Gibbs free energy difference, $\Delta G$, between the liquid and the crystalline phase we use results from differential scanning calorimetry [22] while the interfacial energy $\sigma$ is treated as a fit parameter. The growth velocity, $u=f l^{-1} D_{\text {eff }}\left[1-\exp \left(-\nu_{m} \Delta G / k T\right)\right]$, of the solid phase can similarly be expressed as a product of a kinetic and a thermodynamic factor. For simplicity, we use the same effective diffusivity, $D_{\text {eff }}$, as above and take the fraction of sites at the liquid-crystal interface, where atoms are preferentially added or removed to be $f=1$. Besides $D_{\text {eff }}$, the time [13],

$$
t_{x}=\left(\frac{3 x}{\pi I_{s} u^{3}}\right)^{1 / 4},
$$

to crystallize a small volume fraction $x$ includes only $A$ and $\sigma$ as fit parameters. With the commonly used relation, $D_{\text {eff }} \sim \eta^{-1}$, the minimum in the nucleation times $t_{x}$ at $895 \mathrm{~K}$ leads to an interfacial energy of $\sigma=0.040 \mathrm{Jm}^{-2}$ (solid curve in Fig. 3). While the low temperature part of the crystallization data cannot be described with the assumption $D_{\text {eff }} \sim \eta^{-1}$, for temperatures $T>850 \mathrm{~K}$ satisfactory agreement between the experimental findings and classical nucleation and growth theory is found. In contrast, an Arrhenius-like effective diffusivity $D_{\text {eff }} \sim \exp \left(-Q_{\text {eff }} / k T\right)$, with $Q_{\text {eff }}=1.2 \mathrm{eV}$, describes the crystallization times in the vicinity of the glass transition very well (dashed curve in Fig. 3). 
The temperature dependence of $D_{\text {eff }}$ resembles that of the tracer diffusion of medium sized atoms discussed above, and it is tempting to use a hybrid equation similar to Eq. (3) for $D_{\text {eff }}$. One cannot expect, however, that effective diffusivities and activation energies used to describe kinetics at liquid-solid interfaces during crystallization in a multicomponent system are directly comparable to tracer diffusion coefficients. Highly collective transport mechanisms proposed to control atomic transport in the bulk of the sample are likely to be altered in the immediate vicinity of a crystalline cluster.

We have found that the viscosity of V1 in the equilibrium melt is 3 orders of magnitude larger than for simple metallic systems. The sluggish kinetics reflected in the high viscosity in the temperature above $T_{n}$ contribute significantly to the glass forming ability of the alloy. The time scales obtained from our viscosity measurements suggest that in the deeply supercooled liquid a cascade of transitions from liquidlike to solidlike diffusion of small and medium sized atoms occurs. Similarly, the temperature dependence of the time to crystallization at low temperatures is best described with an Arrhenius-like effective diffusivity. For the present system we therefore conclude that the stability with respect to crystallization in the vicinity of the glass transition is not directly related to the sluggish kinetics at high temperatures that favor glass formation.

This work was supported by the U.S. Department of Energy, Grant No. DEFG-03-86ER45242 and by the ALCOA Technical Center.

*Author to whom correspondence should be addressed. Electronic address: busch@engr.orst.edu

Permanent address: Department of Mechanical Engineering, Oregon State University, Rogers Hall 204, Corvallis, OR 97331-6001.

[1] T. Iida and R. I.L. Guthrie, The Physical Properties of Liquid Metals (Clarendon, Oxford, 1988).

[2] For viscous flow one defines an apparent activation energy as $Q_{\eta}(T)=\partial(\ln \eta) / \partial\left(1 / k_{B} T\right)$ which can be determined from the slope of the logarithm of the viscosity plotted versus inverse temperature.

[3] U. Geyer, S. Schneider, W. L. Johnson, Y. Qiu, T. A. Tombrello, and M. P. Macht, Phys. Rev. Lett. 75, 2364 (1995).
[4] F. Wenwer, K. Knorr, M. P. Macht, and H. Mehrer, Defect Diffus. Forum 143-147, 831 (1997).

[5] E. Budke, P. Fielitz, M. P. Macht, V. Naundorf, and G. Frohberg, Defect Diffus. Forum 143-147, 825 (1997).

[6] H. Ehmler, A. Heesemann, K. Rätzke, F. Faupel, and U. Geyer, Phys. Rev. Lett. 80, 4919 (1998).

[7] E. Bakke, R. Busch, and W. L. Johnson, Appl. Phys. Lett. 67, 3260 (1995).

[8] G. Wilde, G. P. Görler, K. Jeropoulos, R. Willnecker, and H. J. Fecht, Mater. Sci. Forum 269-272, 541 (1998).

[9] A. Waniuk, R. Busch, A. Masuhr, and W. L. Johnson, Acta Mater. 46, 5229 (1998).

[10] R. Busch, S. Schneider, A. Peker, and W. L. Johnson, Appl. Phys. Lett. 67, 1544 (1995).

[11] S. Schneider, P. Thiyagarajan, and W. L. Johnson, Appl. Phys. Lett. 68, 493 (1996).

[12] K. F. Kelton, Philos. Mag. Lett. 77, 337 (1998).

[13] D. R. Uhlmann, J. Non-Cryst. Solids 7, 337 (1972).

[14] A. Masuhr, Ph.D. thesis, California Institute of Technology, 1999.

[15] A. Masuhr, R. Busch, A. Waniuk, and W. L. Johnson, J. Non-Cryst. Solids (to be published).

[16] G. S. Grest and M. H. Cohen, Adv. Chem. Phys. 48, 455 (1981).

[17] K. Ohsaka, S. K. Chung, W. K. Rhim, A. Peker, D. Scruggs, and W. L. Johnson, Appl. Phys. Lett. 70, 726 (1997).

[18] S. Glasstone, K. J. Laidler, and H. Eyring, The Theory of Rate Processes (McGraw-Hill, New York, 1941).

[19] In conjunction with the values for $b$ and $\nu_{m}$ (see below) we find $s_{0}=0.24 \mathrm{eV}^{-3}$. In light of the discussion of Ref. [16], Chap. 3, this is a reasonable value for the temperature independent part of the volume dependence of the free energy of a liquidlike cell.

[20] R. Busch and W. L. Johnson, Mater. Sci. Forum 269-272, 577 (1998).

[21] M. L. William, R. F. Landel, and J. D. Ferry, J. Am. Chem. Soc. 77, 3701 (1955).

[22] R. Busch, Y.J. Kim, and W. L. Johnson, J. Appl. Phys. 77, 4039 (1995).

[23] W. L. Johnson, Mater. Sci. Forum 225-227, 35 (1996).

[24] A. Masuhr and U. Geyer (unpublished).

[25] A. Meyer, J. Wuttke, W. Petry, O.G. Randl, and H. Schober, Phys. Rev. Lett. 80, 4454 (1998).

[26] T.E. Faber, Introduction to the Theory of Liquid Metals (Cambridge University Press, Cambridge, England, 1972).

[27] Y.J. Kim, R. Busch, W. L. Johnson, A. J. Rulison, and W. K. Rhim, Appl. Phys. Lett. 68, 1057 (1996). 